\title{
HARNACK INEQUALITY FOR FRACTIONAL SUB-LAPLACIANS IN CARNOT GROUPS
}

\author{
FAUSTO FERRARI \\ BRUNO FRANCHI
}

\begin{abstract}
In this paper we prove an invariant Harnack inequality on Carnot-Carathéodory balls for fractional powers of sub-Laplacians in Carnot groups. The proof relies on an "abstract" formulation of a technique recently introduced by Caffarelli and Silvestre. In addition, we write explicitly the Poisson kernel for a class of degenerate subelliptic equations in product-type Carnot groups.
\end{abstract}

\section{Contents}

1. Introduction

2. Preliminary results

3. Fractional powers of subelliptic Laplacians

4. Main results

References

\section{INTRODUCTION}

In Euclidean spaces, fractional operators have been studied in connection with different phenomena that can be described as isotropic diffusion with jumps. We mention, for instance, the thin obstacle problem, phase transition problems, and the study of a general class of conformally covariant operators in conformal geometry: see, for instance, [6], 32] and [9]. Typically, these problems can be reduced, in their simplest form, to the study of the equation

$$
(-\Delta)^{\gamma / 2} u=f \quad \text { in } \mathbb{R}^{n}
$$

where $0<\gamma<2$. We remind that the fractional Laplacian in (11) is a nonlocal operator (even more: it is a antilocal operator, see [30]). Nevertheless, solutions of (11) share some properties of the solutions of elliptic equations. More precisely:

- $(-\Delta)^{\gamma / 2}$ is the infinitesimal generator of a Feller semigroup $\left\{T_{t}\right\}_{t>0}$. This means that, if $0 \leq f \leq 1$, then $0 \leq T_{t} f \leq 1$ for $t>0$. By a classical result (see P. Lévy [26], G. A. Hunt [24], Courrège [10] and Bony-Courrège-Priouret [3]), this is equivalent to say that $(-\Delta)^{\gamma / 2}$

1991 Mathematics Subject Classification. 26A33, 35R03 .

Key words and phrases. Carnot groups, heat kernel, fractional powers of sub-Laplacian, Harnack inequality.

The authors are supported by MURST, Italy, and by University of Bologna, Italy, funds for selected research topics and by EC project CG-DICE. 
belongs to a larger class of pseudodifferential operators satisfying the so-called positive maximum principle. We refer to [10 and 3] for an exhaustive discussion; here we restrict ourselves to stress that the positive maximum principle is not the usual maximum principle of potential theory.

- recently, L. Caffarelli \& L. Silvestre [5] proved that functions $u$ that are positive on all of $\mathbb{R}^{n}$ and solve the equation $(-\Delta)^{\gamma / 2} u=0$ in an open set $\Omega \subset \mathbb{R}^{n}$ satisfy an invariant local Harnack inequality. Their technique relies on an extension (or "lifting") procedure, showing ultimately that $u$ can be extended to a function $\tilde{v}$ on $\mathbb{R}^{n+1}$ satisfying a (degenerate) elliptic differential equation.

We remind also that related results have been proved by different methods by N.S. Landkof [25] and K. Bogdan [1].

On the other hand,

- Hunt's theorem in 24] applies to a larger class of differential operators in Lie groups;

- sub-Laplacians in Carnot groups (i.e. in connected and simply connected stratified nilpotent Lie groups) exhibit strong analogies with classical Laplace operator in the Euclidean space (for instance Harnack inequality, maximum principle, existence and estimates of the fundamental solution).

It is therefore natural to ask whether Caffarelli \& Silvestre's approach can be adapted to prove a Harnack inequality for subelliptic fractional equations of the form

$$
\mathcal{L}^{\gamma / 2} u=0,
$$

where $\mathcal{L}$ is a (positive) sublaplacian in a Carnot group $\mathbb{G}$.

In fact, an "abstract" extension technique akin to that of CaffarelliSilvestre has been recently developed in a general setting by Stinga \& Torrea in [35], under very mild hypotheses on the operator $\mathcal{L}$. In particular, they obtained the Harnack inequality for the (fractional) harmonic oscillator. In addition, using analogous arguments, Stinga \& Zhang [36] proved a Harnack inequality for a larger class of fractional operators, containing, for instance, Ornstein-Uhlenbeck operators. However, we stress that subelliptic operators in Carnot groups, though, as a matter of fact, fitting in the wide class of "degenerate elliptic operators", do not belong to the class of degenerate operators considered in [36. Indeed, the degeneration considered in [36] is described by means of $A_{2}$-weights that may vanish only on sets of finite Lebesgue measure. On the contrary, subelliptic Laplacians, when considered as degenerate elliptic operators, may in fact degenerate on all the space. In other words, the degeneration induced by weights is a "degeneration of the measure", whereas subelliptic Laplacians could be considered as LaplaceBeltrami operators for a degenerate geometry.

Typically, if we forget the potentials, operators as in [36] have the form

$$
\left(-\operatorname{div}\left(|x|^{\alpha} \nabla u\right)\right)_{2}^{\gamma / 2}, \quad-n<\alpha<n
$$


in $\mathbb{R}^{n}$, whereas, the simplest instance of our operators is provided by the fractional sub-Laplacian of the first Heisenberg group $\mathbb{H}^{1}$

$$
\left(-\left(\partial_{x}+2 y \partial_{z}\right)^{2} u-\left(\partial_{y}-2 x \partial_{z}\right)^{2} u\right)^{\gamma / 2}
$$

in $\mathbb{R}^{3}$. Some comments in this sense can be found already in [16].

In this paper we further develop the idea of an abstract approach to the problem. However, the setting of Carnot groups, with a natural notion of group convolution, makes possible to recover, starting from the abstract representation in terms of the spectral resolution, another explicit form of the fractional powers (in terms of convolutions with singular kernels), as well as of the lifting operator (in terms of the convolution with a suitable Poisson kernel).

We like also to mention that, in the special case of Heisenberg groups, an explicit representation of the Poisson kernel is given also in [21] through different methods (group Fourier transform).

To state our main result, we need preliminarily to remind that in any Carnot group we can define a left-invariant distance $d_{c}$ (the so-called CarnotCarathéodory distance) that fits the structure of the group. If we denote by $B_{c}=B_{c}(x, r)(x \in \mathbb{G}$ and $r>0)$ the metric balls associated with $d_{c}$ and by $W_{\mathbb{G}}^{s, 2}$ the Folland-Stein Sobolev space in $\mathbb{G}$ (see Section 1 for details), then the Harnack inequality for fractional sub-Laplacians takes an invariant intrinsic form. More precisely, we have:

Theorem Let $-1<a<1$ and let $u \in W_{\mathbb{G}}^{1-a, 2}(\mathbb{G})$ be given, $u \geq 0$ on all of $\mathbb{G}$. Assume $\mathcal{L}^{(1-a) / 2} u=0$ in an open set $\Omega \subset \mathbb{G}$.

Then there exist $C, b>0$ (independent of $u$ ) such that the following invariant Harnack inequality holds:

$$
\sup _{B_{c}(x, r)} u \leq C \inf _{B_{c}(x, r)} u
$$

for any metric ball $B_{c}(x, r)$ such that $B_{c}(x, b r) \subset \Omega$.

Let us sketch briefly the main features of our proof. Basically, still following [5, its core consists in the construction of a $\mathcal{L}$-harmonic "lifting" operator $u=u(x) \rightarrow v=v(x, y)$ from $\mathbb{G}$ to $\mathbb{G} \times \mathbb{R}^{+}$by means of the spectral resolution of $\mathcal{L}$ in $L^{2}(\mathbb{G})$ in such a way that $u$ is the trace of the normal derivative of $v$ on $y=0$. If, in particular, $a=0$, then this operator is nothing but the semigroup generated by $-\mathcal{L}^{1 / 2}$.

Subsequently, as in [5], we show that, if $\mathcal{L}^{\frac{1-a}{2}} u=0$ in an open set $\Omega$ then its lifting $v$ can be continued by parity across $y=0$ to a weak solution $\tilde{v}$ of the equation

$$
\tilde{\mathcal{L}} \tilde{v}:=-|y|^{a} \mathcal{L} \tilde{v}+\partial_{y}\left(|y|^{a} \partial_{y} \tilde{v}\right)=0 .
$$

In addition we show that the lifting operator can be also written as a convolution operator with a positive kernel $P_{\mathbb{G}}$, that is written explicitly. Thus $\tilde{v} \geq 0$ if $u \geq 0$ on all $\mathbb{G}$, and therefore our problem reduces to prove Harnack inequality for a weighted sub-elliptic differential operator. The construction of $P_{\mathbb{G}}$ not only yields the possibility of replacing the assumption $u \in W_{\mathbb{G}}^{1-a, 2}(\mathbb{G})$ by some weaker assumptions on the behavior of $u$ at infinity (in the spirit of some remarks in [5]), but provides an explicit form for the 
Poisson kernel $P_{\mathbb{G}}(\cdot, y)$ in the half-space $\mathbb{G} \times(0, \infty)$ for $\tilde{\mathcal{L}}$. More precisely, if we denote by $h(t, \cdot)$ the heat kernel associated with $-\mathcal{L}$ as in [13], then

$$
P_{\mathbb{G}}(\cdot, y):=C_{a} y^{1-a} \int_{0}^{\infty} t^{(a-3) / 2} e^{-\frac{y^{2}}{4 t}} h(t, \cdot) d t,
$$

where

$$
C_{a}=2^{a-1} \Gamma((1-a) / 2)^{-1} .
$$

A similar formula appears in 35, but, as long as we know, this representation is new for sublaplacians in Carnot groups.

The paper is organized as follows: in Section 2 we fix our notations for Carnot groups and for Harnack inequality in this setting; in Section 3 we collect some more or less known results on fractional powers of sub-Laplacian in Carnot groups and we prove different representation theorems. Finally, in Section 4 we prove our main results.

\section{Preliminary Results}

A connected and simply connected Lie group $(\mathbb{G}, \cdot)$ (in general non-commutative) is said a Carnot group of step $\kappa$ if its Lie algebra $\mathfrak{g}$ admits a step $\kappa$ stratification, i.e. there exist linear subspaces $V_{1}, \ldots, V_{\kappa}$ such that

$$
\mathfrak{g}=V_{1} \oplus \ldots \oplus V_{\kappa}, \quad\left[V_{1}, V_{i}\right]=V_{i+1}, \quad V_{\kappa} \neq\{0\}, \quad V_{i}=\{0\} \text { if } i>\kappa,
$$

where $\left[V_{1}, V_{i}\right]$ is the subspace of $\mathfrak{g}$ generated by the commutators $[X, Y]$ with $X \in V_{1}$ and $Y \in V_{i}$. The first layer $V_{1}$, the so-called horizontal layer, plays a key role in the theory, since it generates $\mathfrak{g}$ by commutation.

For a general introduction to Carnot groups from the point of view of the present paper, we refer, e.g., to 2, [14 and 34.

Set $m_{i}=\operatorname{dim}\left(V_{i}\right)$, for $i=1, \ldots, \kappa$ and $h_{i}=m_{1}+\cdots+m_{i}$, so that $h_{\kappa}=n$. For sake of simplicity, we write also $m:=m_{1}$. We denote by $Q$ the homogeneous dimension of $\mathbb{G}$, i.e. we set

$$
Q:=\sum_{i=1}^{\kappa} i \operatorname{dim}\left(V_{i}\right)
$$

If $e$ is the unit element of $(\mathbb{G}, \cdot)$, we remind that the map $X \rightarrow X(e)$, that associate with a left-invariant vector field $X$ its value at $e$, is an isomorphism from $\mathfrak{g}$ to $T \mathbb{G}_{e}$, in turn identified with $\mathbb{R}^{n}$. From now on, we shall use systematically these identications. Thus, the horizontal layer defines, by left translation, a fiber bundle $H \mathbb{G}$ over $\mathbb{G}$ (the horizontal bundle). Its sections are the horizontal vector fields.

We choose now a basis $e_{1}, \ldots, e_{n}$ of $\mathbb{R}^{n}$ adapted to the stratification of $\mathfrak{g}$, i.e. such that

$$
e_{h_{j-1}+1}, \ldots, e_{h_{j}} \text { is a basis of } V_{j} \text { for each } j=1, \ldots, \kappa .
$$

Then, we denote by $\langle\cdot, \cdot\rangle$ the scalar product in $\mathfrak{g}$ making the adapted basis $\left\{e_{1}, \ldots, e_{n}\right\}$ orthonormal. Moreover, let $X=\left\{X_{1}, \ldots, X_{n}\right\}$ be the family of left invariant vector fields such that $X_{i}(e)=e_{i}, i=1, \ldots, n$. Clearly, $X$ is othonormal with respect to $\langle\cdot, \cdot\rangle$.

A Carnot group $\mathbb{G}$ can be always identified, through exponential coordinates, with the Euclidean space $\left(\mathbb{R}^{n}, \cdot\right)$, where $n$ is the dimension of $\mathfrak{g}$, 
endowed with a suitable group operation. The explicit expression of the group operation - is determined by the Campbell-Hausdorff formula.

For any $x \in \mathbb{G}$, the (left) translation $\tau_{x}: \mathbb{G} \rightarrow \mathbb{G}$ is defined as

$$
z \mapsto \tau_{x} z:=x \cdot z .
$$

For any $\lambda>0$, the dilation $\delta_{\lambda}: \mathbb{G} \rightarrow \mathbb{G}$, is defined as

$$
\delta_{\lambda}\left(x_{1}, \ldots, x_{n}\right)=\left(\lambda^{d_{1}} x_{1}, \ldots, \lambda^{d_{n}} x_{n}\right),
$$

where $d_{i} \in \mathbb{N}$ is called homogeneity of the variable $x_{i}$ in $\mathbb{G}$ (see [14 Chapter 1) and is defined as

$$
d_{j}=i \quad \text { whenever } h_{i-1}+1 \leq j \leq h_{i},
$$

hence $1=d_{1}=\ldots=d_{m_{1}}<d_{m_{1}+1}=2 \leq \ldots \leq d_{n}=\kappa$.

Through this paper, by $\mathbb{G}$-homogeneity we mean homogeneity with respect to group dilations $\delta_{\lambda}$ (see again [14] Chapter 1).

The Haar measure of $\mathbb{G}=\left(\mathbb{R}^{n}, \cdot\right)$ is the Lebesgue measure in $\mathbb{R}^{n}$. If $A \subset \mathbb{G}$ is $L$-measurable, we write $|A|$ to denote its Lebesgue measure. Moreover, if $m \geq 0$, we denote by $\mathcal{H}^{m}$ the $m$-dimensional Hausdorff measure obtained from the Euclidean distance in $\mathbb{R}^{n} \simeq \mathbb{G}$.

The following result is contained in [14, Proposition 1.26.

Proposition 2.1. If $j=1, \ldots, m$, the vector fields $X_{j}$ have polynomial coefficients and have the form

$$
X_{j}(x)=\partial_{j}+\sum_{d_{k}>1}^{n} p_{j, k}(x) \partial_{k},
$$

where the $p_{j, k}$ are $\mathbb{G}$-homogeneous polynomials of degree $d_{k}-1$ for $d_{k}>1$.

Once a basis $X_{1}, \ldots, X_{m}$ of the horizontal layer is fixed, we define, for any function $f: \mathbb{G} \rightarrow \mathbb{R}$ for which the partial derivatives $X_{j} f$ exist, the horizontal gradient of $f$, denoted by $\nabla_{\mathbb{G}} f$, as the horizontal section

$$
\nabla_{\mathbb{G}} f:=\sum_{i=1}^{m}\left(X_{i} f\right) X_{i},
$$

whose coordinates are $\left(X_{1} f, \ldots, X_{m} f\right)$. Moreover, if $\phi=\left(\phi_{1}, \ldots, \phi_{m}\right)$ is an horizontal section such that $X_{j} \phi_{j} \in L_{\text {loc }}^{1}(\mathbb{G})$ for $j=1, \ldots, m$, we define $\operatorname{div}_{\mathbb{G}} \phi$ as the real valued function

$$
\operatorname{div}_{\mathbb{G}}(\phi):=-\sum_{j=1}^{m} X_{j}^{*} \phi_{j}=\sum_{j=1}^{m} X_{j} \phi_{j} .
$$

Following [14, we also adopt the following multi-index notation for higherorder derivatives. If $I=\left(i_{1}, \ldots, i_{n}\right)$ is a multi-index, we set $X^{I}=X_{1}^{i_{1}} \cdots X_{n}^{i_{n}}$. By the Poincaré-Birkhoff-Witt theorem (see, e.g. 4, I.2.7), the differential operators $X^{I}$ form a basis for the algebra of left invariant differential operators in $\mathbb{G}$. Furthermore, we set $|I|:=i_{1}+\cdots+i_{n}$ the order of the differential operator $X^{I}$, and $d(I):=d_{1} i_{1}+\cdots+d_{n} i_{n}$ its degree of $\mathbb{G}$-homogeneity with respect to group dilations. 
Let $X_{1}, \ldots, X_{m}$ be a basis of the first layer of $\mathfrak{g}$, we denote by $\mathcal{L}$ the associated positive sub-Laplacian

$$
\mathcal{L}:=-\sum_{j=1}^{m} X_{j}^{2}
$$

It is easy to see that

$$
\mathcal{L} u=-\operatorname{div}_{\mathbb{G}}\left(\nabla_{\mathbb{G}} u\right) .
$$

In addition, $\mathcal{L}$ is left-invariant, i.e. for any $x \in \mathbb{G}$, we have

$$
\mathcal{L}\left(u \circ \tau_{x}\right)=(\mathcal{L} u) \circ \tau_{x} .
$$

Following e.g. [14, we can define a group convolution in $\mathbb{G}$ : if, for instance, $f \in \mathcal{D}(\mathbb{G})$ and $g \in L_{\text {loc }}^{1}(\mathbb{G})$, we set

$$
f * g(x):=\int f(y) g\left(y^{-1} x\right) d y \quad \text { for } x \in \mathbb{G} .
$$

We remind that, if (say) $g$ is a smooth function and $L$ is a left invariant differential operator, then $L(f * g)=f * L g$. We remind also that the convolution is again well defined when $f, g \in \mathcal{D}^{\prime}(\mathbb{G})$, provided at least one of them has compact support (as customary, we denote by $\mathcal{E}^{\prime}(\mathbb{G})$ the class of compactly supported distributions in $\mathbb{G}$ identified with $\mathbb{R}^{n}$ ).

If $E \subset \mathbb{G}$ is a mesurable set, a notion of $\mathbb{G}$-perimeter measure $|\partial E|_{\mathbb{G}}$ has been introduced in [20. We refer to [20], [17, [19], 18] for a detailed presentation. For our needs, we restrict ourselves to remind that, if $E$ has locally finite $\mathbb{G}$-perimeter (is a $\mathbb{G}$-Caccioppoli set), then $|\partial E|_{\mathbb{G}}$ is a Radon measure in $\mathbb{G}$, invariant under group translations and $\mathbb{G}$-homogeneous of degree $Q-1$. Moreover, the following representation theorem holds (see [8]).

Proposition 2.2. If $E$ is a $\mathbb{G}$-Caccioppoli set with Euclidean $\mathbf{C}^{1}$ boundary, then there is an explicit representation of the $\mathbb{G}$-perimeter in terms of the Euclidean $(n-1)$-dimensional Hausdorff measure $\mathcal{H}^{n-1}$

$$
|\partial E|_{\mathbb{G}}(\Omega)=\int_{\partial E \cap \Omega}\left(\sum_{j=1}^{m_{1}}\left\langle X_{j}, n\right\rangle_{\mathbb{R}^{n}}^{2}\right)^{1 / 2} d \mathcal{H}^{n-1},
$$

where $n=n(x)$ is the Euclidean unit outward normal to $\partial E$.

We have also

Proposition 2.3. If $E$ is a regular bounded open set with Euclidean $\mathbf{C}^{1}$ boundary and $\phi$ is a horizontal vector field, continuously differentiable on $\bar{\Omega}$, then

$$
\int_{E} \operatorname{div}_{\mathbb{G}} \phi d x=\int_{\partial E}\left\langle\phi, n_{\mathbb{G}}\right\rangle d|\partial E|_{\mathbb{G}},
$$

where $n_{\mathbb{G}}(x)$ is the intrinsic horizontal outward normal to $\partial E$, given by the (normalized) projection of $n(x)$ on the fiber $H \mathbb{G}_{x}$ of the horizontal fibre bundle $H \mathbb{G}$.

Remark 2.4. The definition of $n_{\mathbb{G}}$ is well done, since $H \mathbb{G}_{x}$ is transversal to the tangent space to $E$ at $x$ for $|\partial E|_{\mathbb{G}}$-a.e. $x \in \partial E$ (see [29]). 
Definition 2.5. (Carnot-Carathéodory distance) An absolutely continuous curve $\gamma:[0, T] \rightarrow \mathbb{G}$ is a sub-unit curve with respect to $X_{1}, \ldots, X_{m}$ if it is an horizontal curve, i.e. if there are real measurable functions $c_{1}(s), \ldots, c_{m}(s), s \in[0, T]$ such that

$$
\dot{\gamma}(s)=\sum_{j=1}^{m} c_{j}(s) X_{j}(\gamma(s)), \quad \text { for a.e. } s \in[0, T],
$$

and if, in addition,

$$
\sum_{j} c_{j}^{2} \leq 1
$$

If $x, y \in \mathbb{G}$, their Carnot-Carathéodory distance (cc-distance) $d_{c}(x, y)$ is defined as follows:

$d_{c}(x, y)=\inf \{T>0:$ there is a subunit curve $\gamma$ with $\gamma(0)=x, \gamma(T)=y\}$.

The set of subunit curves joining $x$ and $y$ is not empty, by Chow's theorem, since by (2), the rank of the Lie algebra generated by $X_{1}, \ldots, X_{m}$ is $n$; hence $d_{c}$ is a distance on $\mathbb{G}$ inducing the same topology as the standard Euclidean distance. We shall denote $B_{c}(x, r)$ the open balls associated with $d_{c}$. The cc-distance is well behaved with respect to left translations and dilations, that is

$$
d_{c}(z \cdot x, z \cdot y)=d_{c}(x, y) \quad, \quad d_{c}\left(\delta_{\lambda}(x), \delta_{\lambda}(y)\right)=\lambda d_{c}(x, y)
$$

for $x, y, z \in \mathbb{G}$ and $\lambda>0$.

We have also

$$
\left|B_{c}(x, r)\right|=r^{Q}\left|B_{c}(0,1)\right| \text { and }\left|\partial B_{c}(x, r)\right|_{\mathbb{G}}(\mathbb{G})=r^{Q-1}\left|\partial B_{c}(0,1)\right|_{\mathbb{G}}(\mathbb{G}) .
$$

Denote by $Y$ the vector field $\frac{\partial}{\partial y}$ in $\hat{\mathbb{G}}:=\mathbb{G} \times \mathbb{R}$. The Lie group $\hat{\mathbb{G}}$ is a Carnot group; its Lie algebra $\hat{\mathfrak{g}}$ admits the stratification

$$
\hat{\mathfrak{g}}=\hat{V}_{1} \oplus V_{2} \oplus \cdots \oplus V_{\kappa},
$$

where $\hat{V}_{1}=\operatorname{span}\left\{Y, V_{1}\right\}$. Since the basis $\left\{X_{1}, \ldots, X_{m}\right\}$ of $V_{1}$ has been already fixed once and for all, the associated basis for $\hat{V}_{1}$ will be $\left\{X_{1}, \ldots, X_{m}, Y\right\}$.

The following statement follows trivially from the definition of CarnotCarathéodory distance, keeping into account that the coefficients of $X_{1}, \cdots, X_{m}$ in $\hat{\mathbb{G}}$ are independent of $y$.

Lemma 2.6. Denote by $\hat{B}_{c}((x, y), r)$ a Carnot-Carathéodory ball in $\hat{\mathbb{G}}$ centered at the point $(x, y) \in \hat{\mathbb{G}}$ and $B_{c}(x, r)$ the Carnot-Carathéodory ball in $\mathbb{G}$ centered at the point $x \in \mathbb{G}$. Then

$$
\hat{B}_{c}((x, 0), r) \cap\{y=0\}=B_{c}(x, r) \times\{0\} .
$$

Moreover, if $(x, y) \in K$, where $K \subset \mathbb{G} \times \mathbb{R}$ is a compact set, and $r \leq r_{0}$ there exist $\sigma_{1}, \sigma_{2}>0$ (independent of $r$ and $(x, y)$ ) such that

$$
\hat{B}_{c}\left((x, y), \sigma_{1} r\right) \subset B_{c}(x, r) \times(y-r, y+r) \subset \hat{B}_{c}\left((x, y), \sigma_{2} r\right) .
$$

Definition 2.7 (see [31, [7]). A function $\omega \in L_{\text {loc }}^{1}(\mathbb{G})$ is said to be a $A_{2^{-}}$ weight with respect to the cc-metric of $\mathbb{G}$ if

$$
\sup _{x \in \mathbb{G}, r>0} f_{B_{c}(x, r)} \omega(y) d y \cdot f_{7} \omega(y)^{-1} d y<\infty .
$$


The following remark will be crucial in Section 4 .

Remark 2.8. By Lemma 2.6, the function $\omega(x, y)=|y|^{a}$ is a $A_{2}$-weight with respect to the cc-metric of $\mathbb{G} \times \mathbb{R}$ if and only if $-1<a<1$.

The following result, that is the counterpart in the sub-elliptic framework of the Euclidean setting (see e.g. [11] and [33]), can be found in [28]. This idea goes back (at least for the so-called "Grushin type" vector fields) to [15] and [16]. Basically, this is possible thanks to weighted Sobolev-Poincaré inequalities in Carnot groups.

For further results concerning the boundary Harnack principle in Carnot groups we refer to [12].

Theorem 2.9. Let $\mathbb{G}$ be a Carnot group, and let $\Omega \subset \mathbb{G}$ be an open set. Let now $\omega \in L_{\text {loc }}^{1}(\mathbb{G})$ be a $A_{2}$-weight with respect to the Carnot-Carathéodory metric $d_{c}$ of $\mathbb{G}$. Then, if $u \in W_{\mathbb{G}}^{1,2}(\Omega, \omega d x)$ is a weak solution of

$$
\operatorname{div}_{\mathbb{G}}\left(\omega \nabla_{\mathbb{G}} u\right)=0,
$$

then $u$ is locally Hölder continuous in $\Omega$. If, in addition, $u \geq 0$, then there exist $C, b>0$ (independent of $u$ ) such that the following invariant Harnack inequality holds:

$$
\sup _{B_{c}(x, r)} u \leq C \inf _{B_{c}(x, r)} u
$$

for any metric ball $B_{c}(x, r)$ such that $B_{c}(x, b r) \subset \Omega$.

Suppose now $\Omega$ satisfies the following local condition (S): for any $x_{0} \in \partial \Omega$ there exist $r_{0}>0$ and $\alpha>0$ such that

$$
\left|B_{c}\left(x_{0}, r\right) \cap \Omega^{c}\right| \geq \alpha\left|B_{c}\left(x_{0}, r\right)\right| \text { for } r<r_{0} .
$$

Then $u$ is locally Hölder continuous in $\bar{\Omega}$.

\section{Fractional powers of SUbelliptic Laplacians}

Definition 3.1. Let $\alpha \in \mathbb{C}$. We call $K_{\alpha}$ a kernel of type $\alpha$ (according to Folland) a distribution which is smooth away from 0 and $\mathbb{G}$-homogeneus of degree $\alpha-Q$.

Remark 3.2. Let $K_{\alpha}$ be a positive kernel of type $\alpha$; then there exist $m, M \in$ $\mathbb{R}$, with $0<m \leq M<\infty$, such that

$$
m d(y, 0)^{\alpha-Q}<K_{\alpha}(y)<M d(y, 0)^{\alpha-Q},
$$

for any $y \in \mathbb{G}$.

Proposition 3.3. Suppose $0<\beta<Q$. Denote by $h=h(t, x)$ the fundamental solution of $\mathcal{L}+\partial / \partial t$ (see [13], Proposition 3.3). Then the integral

$$
R_{\beta}(x)=\frac{1}{\Gamma(\beta / 2)} \int_{0}^{\infty} t^{\frac{\beta}{2}-1} h(t, x) d t
$$

converges absolutely for $x \neq 0$. In addition, $R_{\beta}$ is a kernel of type $\beta$.

Moreover

i) $R_{2}$ is the fundamental solution of $\mathcal{L}$;

ii) if $\alpha \in(0,2)$ and $u \in \mathcal{D}(\mathbb{G})$, then

$$
\mathcal{L}^{\alpha / 2} u=\mathcal{L} u * R_{2-\alpha} .
$$


iii) the kernels $R_{\alpha}$ admit the following convolution rule: if $\alpha>0, \beta>0$ and $x \neq 0$, then

$$
R_{\alpha+\beta}(x)=R_{\alpha}(x) * R_{\beta}(x) .
$$

Proof. These results are basically contained in [13]. Let us sketch the proof of ii): by [13], Theorem 3.15, iii), and Proposition 3.18, keeping in mind that $\mathcal{D}(\mathbb{G})$ is contained in the domain of all real powers of $\mathcal{L}$, we obtain

$$
\mathcal{L}^{\alpha / 2} u=\mathcal{L}^{(\alpha-2) / 2} \mathcal{L} u=\mathcal{L} u * R_{2-\alpha} .
$$

Remark 3.4. If $\beta<0, \beta \notin\{0,-2,-4, \cdots\}$, then again

$$
\widetilde{R}_{\beta}(x)=\frac{\frac{\beta}{2}}{\Gamma(\beta / 2)} \int_{0}^{\infty} t^{\frac{\beta}{2}-1} h(t, x) d t
$$

defines a smooth function in $\mathbb{G} \backslash\{0\}$, since $t \rightarrow h(t, x)$ vanishes of infinite order as $t \rightarrow 0$ if $x \neq 0$. In addition, $\widetilde{R}_{\beta}$ is positive and $\mathbb{G}$-homogeneous of degree $\beta-Q$. However, unlike $R_{\beta}$ for $0<\beta<Q, \widetilde{R}_{\beta}$ is not a kernel of type $\beta$, since it does not belong to $L_{\text {loc }}^{1}(\mathbb{G})$. Integrating by parts, it is easy to see also that, if $0<\alpha<2$, then

$$
\mathcal{L} R_{2-\alpha}=\widetilde{R}_{-\alpha}
$$

for $x \neq 0$.

Definition 3.5. We set (we remind that $R_{\beta}>0$ for $0<\beta<Q$ )

$$
\rho(x)=R_{2-\alpha}^{1 /(2-\alpha-Q)} .
$$

It is easy to see that $\rho$ is an $\mathbb{G}$-homogeneous norm in $\mathbb{G}$, smooth outside of the origin. In addition, $d(x, y):=\rho\left(y^{-1} x\right)$ is a quasi-distance in $\mathbb{G}$. In turn, $d$ is equivalent to the Carnot-Carathéodory distance on $G$, as well as to any other $\mathbb{G}$-homogeneous left invariant distance on $\mathbb{G}$.

Proposition 3.6. Denote by $B_{\rho}=B_{\rho}(x, r)$ the metric balls given by $\rho$. We have:

$$
\begin{gathered}
m d_{c}(x, y) \leq d(x, y) \leq M d_{c}(x, y) \quad \text { for all } x, y \in \mathbb{G} ; \\
m r^{Q} \leq\left|B_{\rho}(x, r)\right| \leq M r^{Q} ; \\
m r^{Q-1} \leq \mathcal{H}_{\mathbb{G}}^{Q-1}\left(\partial B_{\rho}(x, r)\right) \leq M r^{Q-1} .
\end{gathered}
$$

Definition 3.7. We denote by $x \rightarrow{ }^{\mathrm{w}} x$ the "semicheck" map

$$
\left(x_{1}, \ldots, x_{n}\right) \rightarrow\left((-1)^{d_{1}} x_{1},(-1)^{d_{2}} x_{2}, \ldots,(-1)^{d_{n}} x_{n}\right) .
$$

From now on, we adopt the following notation: ${ }^{\mathrm{w}} f(x, t):=f\left({ }^{\mathrm{w}} x, t\right)$ for any function $f$ defined in $\mathbb{G} \times \mathbb{R}$.

Theorem 3.8. We have:

i) if $j=1, \ldots, m$, then $X_{j}{ }^{\mathrm{w}}=-{ }^{\mathrm{w}} X_{j}$. In particular, $\mathcal{L}^{\mathrm{w}}={ }^{\mathrm{w}} \mathcal{L}$;

ii) if $h$ is the fundamental solution of $\partial_{t}+\mathcal{L}$, then ${ }^{\mathrm{w}} h=h$;

iii) if $\alpha>0, R_{\alpha}={ }^{\mathrm{w}} R_{\alpha}$ and $\tilde{R}_{-\alpha}={ }^{\mathrm{w}} \tilde{R}_{-\alpha}$. In particular, ${ }^{\mathrm{w}} \rho=\rho$;

iv) $d_{c}\left({ }^{\mathrm{w}} x,{ }^{\mathrm{w}} y\right)=d_{c}(x, y)$ for all $x, y \in \mathbb{G}$. 
v) if $E \subset \mathbb{G}$ is a $\mathbb{G}$-Cacciopoli set, then the perimeter measure $|\partial E|_{\mathbb{G}}$ is semicheck-invariant.

Proof. The core of the proof relies in the following identity. If $p_{j k}$ are the polynomials defined in Proposition 2.1, then

$$
p_{j, k}\left({ }^{\mathrm{w}} x\right)=(-1)^{d_{k}-1} p_{j, k}(x) .
$$

To prove (13), we remind that $p_{j, k}$ is a $\mathbb{G}$-homogeneous polynomials of degree $d_{k}-1$. Let now $\alpha=\left(\alpha_{1}, \ldots, \alpha_{n}\right)$ be a multi-index, and let $x^{\alpha}$ be an arbitrary $\mathbb{G}$-homogeneous monomial of degree $d_{k}-1$, i.e. assume

$$
d_{1} \alpha_{1}+\cdots+d_{n} \alpha_{n}=d_{k}-1 .
$$

We have but to show that (13) holds for $x^{\alpha}$.

If $\ell=1, \ldots, n$, we set $I_{\ell}:=\left\{i ; d_{i}=\ell\right\}$. Gathering in (14) the terms with $d_{i}=\ell$, identity (14) becomes

$$
\sum_{\ell} d_{\ell}\left(\sum_{i \in I_{\ell}} \alpha_{i}\right)=d_{k}-1 .
$$

Then

$$
\left({ }^{\mathrm{w}} x\right)^{\alpha}=(-1)^{\sum_{\ell} d_{\ell}\left(\sum_{i \in I_{\ell}} \alpha_{i}\right)} x^{\alpha},
$$

and the assertion follows by (15).

Let us prove now i). If $u$ is a (say) smooth function, by (13), we have

$$
\begin{aligned}
X_{j}\left({ }^{\mathrm{w}} u(x)\right)=X_{j}\left(u\left({ }^{\mathrm{w}} x\right)\right) \\
\quad=-\left(\partial_{j} u\right)\left({ }^{\mathrm{w}} x\right)+\sum_{d_{k}>1} p_{j, k}(x)(-1){ }^{d_{k}}\left(\partial_{k} u\right)\left({ }^{\mathrm{w}} x\right) \\
\quad=-\left(\partial_{j} u\right)\left({ }^{\mathrm{w}} x\right)-\sum_{d_{k}>1} p_{j, k}\left({ }^{\mathrm{w}} x\right)\left(\partial_{k} u\right)\left({ }^{\mathrm{w}} x\right) \\
\quad=-\left(X_{j} u\right)\left({ }^{\mathrm{w}} x\right)=-{ }^{\mathrm{w}}\left(X_{j} u\right)(x) .
\end{aligned}
$$

In order to prove ii), let us show preliminarily that $h\left(t,{ }^{\mathrm{w}} x\right)$ is still a fundamental solution of $\partial_{t}+\mathcal{L}$. Indeed, if $u \in \mathcal{D}(\mathbb{R} \times \mathbb{G})$, we have

$$
\begin{aligned}
& \left\langle\left(\partial_{t}+\mathcal{L}\right) h\left(t,{ }^{\mathrm{w}} x\right) \mid u(t, x)\right\rangle=\left\langle h\left(t,{ }^{\mathrm{w}} x\right) \mid\left(-\partial_{t}+\mathcal{L}\right) u(t, x)\right\rangle \\
& =\left\langle\left. h\right|^{\mathrm{w}}\left(-\partial_{t}+\mathcal{L}\right) u\right\rangle=\left\langle h \mid\left(-\partial_{t}+\mathcal{L}\right)^{\mathrm{w}} u\right\rangle \\
& =\left\langle\left.\left(\partial_{t}+\mathcal{L}\right) h\right|^{\mathrm{w}} u\right\rangle={ }^{\mathrm{w}} u(0,0)=u(0,0) .
\end{aligned}
$$

Therefore, the function

$$
h_{0}:=h-h^{\mathrm{w}}
$$

vanishes at $t=0$ and solves $\left(\partial_{t}+\mathcal{L}\right) h_{0}=0$, being in particular smooth in $\mathbb{R} \times \mathbb{G}$, by the hypoellipticity of $\partial_{t}+\mathcal{L}([23])$. By [13], Corollary 3.5, $h_{0}(t, x) \rightarrow 0$ as $x \rightarrow \infty$ uniformly for $t$ in a bounded interval. Thus we can apply the standard "parabolic" maximum principle to conclude that $h_{0} \equiv 0$, and then ii) follows.

The proof of iii) is straightforward. To prove iv), it is enough to show that, if $x, y \in \mathbb{G}$ and $\gamma$ is a horizontal curve joining $x$ and $y$ with sub-Riemannian length $\ell(\gamma)$, then ${ }^{\mathrm{w}} \gamma$ is still horizontal, $\ell\left({ }^{\mathrm{w}} \gamma\right)=\ell(\gamma)$, and, obviously, joins $x$ and $y$. 
By assumption, we can write

$$
\gamma^{\prime}(t)=\sum_{j=1}^{m} a_{j}(t) X_{j}(\gamma(t)), \quad t \in[0,1]
$$

i.e, if for any $p \in \mathbb{G}$ we write $p_{\ell}$ for the $\ell$-th component of $p$ in exponential coordinates, for $\ell=1,2, \ldots, n$, then

$$
\gamma_{\ell}^{\prime}=\sum_{j=1}^{m} a_{j}\left(X_{j}(\gamma)\right)_{\ell}=\sum_{j=1}^{m} a_{j}\left(e_{j}+\sum_{d_{k}>1} p_{j, k}(\gamma) e_{k}\right)_{\ell},
$$

with

$$
\int_{0}^{1}\left(\sum_{j} a_{j}^{2}(t)\right)^{1 / 2} d t=\ell(\gamma)
$$

Notice that (16) reads as follows:

$$
\gamma_{\ell}^{\prime}=\left\{\begin{array}{lr}
a_{\ell} & \text { if } 1 \leq \ell \leq m \\
\sum_{j} a_{j} p_{j, \ell}(\gamma) & \text { if } \ell>m
\end{array}\right.
$$

Our assertion will follow by showing that

$$
\left({ }^{\mathrm{w}} \gamma\right)^{\prime}(t)=-\sum_{j=1}^{m} a_{j}(t) X_{j}\left({ }^{\mathrm{w}} \gamma(t)\right), \quad t \in[0,1],
$$

Indeed, by (13),

$$
\begin{aligned}
& X_{j}\left({ }^{\mathrm{w}} \gamma\right)=e_{j}+\sum_{d_{k}>1} p_{j, k}\left({ }^{\mathrm{w}} \gamma\right) e_{k} \\
& =(-1)^{d_{1}-1} e_{j}+\sum_{d_{k}>1}(-1)^{d_{k}-1} p_{j, k}(\gamma) e_{k},
\end{aligned}
$$

so that, keeping in mind (17),

$$
\left(\sum_{j=1}^{m} a_{j} X_{j}\left({ }^{\mathrm{w}} \gamma\right)\right)_{\ell}=\left\{\begin{array}{lr}
-(-1)^{d_{1}} a_{\ell}=-\left({ }^{\mathrm{w}} \gamma\right)_{\ell} & \text { if } 1 \leq \ell \leq m \\
-(-1)^{d_{\ell}} \sum_{j} a_{j} p_{j, \ell}(\gamma)=-\left({ }^{\mathrm{w}} \gamma\right)_{\ell} & \text { if } \ell>m .
\end{array}\right.
$$

This proves (18) and achieves the proof of the theorem, since v) is a straightforward consequence of i).

Corollary 3.9. If $\alpha>0$ and $j=1, \ldots, m$, then

$$
{ }^{\mathrm{w}}\left(X_{j} R_{\alpha}\right)=-X_{j} R_{\alpha} \quad \text { and }{ }^{\mathrm{w}}\left(X_{j} \tilde{R}_{-\alpha}\right)=-X_{j} \tilde{R}_{-\alpha} .
$$

We follow the guidelines of [13], Section 3. We have:

Theorem 3.10. The operator $\mathcal{L}$ is a positive self-adjoint operator with domain $W_{\mathbb{G}}^{2,2}(\mathbb{G})$. Denote now by $\{E(\lambda)\}$ the spectral resolution of $\mathcal{L}$ in $L^{2}(\mathbb{G})$. If $\alpha>0$ then

with domain

$$
\mathcal{L}^{\alpha / 2}=\int_{0}^{+\infty} \lambda^{\alpha / 2} d E(\lambda)
$$

$$
W_{\mathbb{G}}^{\alpha, 2}(\mathbb{G}):=\left\{u \in L^{2}(\mathbb{G}): \int_{11}^{+\infty} \lambda^{\alpha} d\langle E(\lambda) u, u\rangle<\infty\right\},
$$


endowed with the graph norm.

Theorem 3.11. If $u \in \mathcal{S}(\mathbb{G})$, and $0<\alpha<2$, then $\mathcal{L}^{\alpha / 2} u \in L^{2}(\mathbb{G})$, and

$$
\begin{aligned}
\mathcal{L}^{\alpha / 2} u(x) & =\int_{\mathbb{G}}\left(u(x y)-u(x)-\omega(y)\left\langle\nabla_{\mathbb{G}} u(x), y\right\rangle\right) \widetilde{R}_{-\alpha}(y) d y \\
& =\text { P.V. } \int_{\mathbb{G}}(u(y)-u(x)) \widetilde{R}_{-\alpha}\left(y^{-1} x\right) d y,
\end{aligned}
$$

where $\omega$ is the characteristic function of the unit ball $B_{\rho}(0,1)$.

Proof. First of all, we notice that the map

$$
y \rightarrow\left(u(x y)-u(x)-\omega(y)\left\langle\nabla_{\mathbb{G}} u(x), y\right\rangle\right) \widetilde{R}_{-\alpha}(y)
$$

belongs to $L^{1}(\mathbb{G})$. Indeed,

$$
\left(u(x y)-u(x)-\omega(y)\left\langle\nabla_{\mathbb{G}} u(x), y\right\rangle\right) \widetilde{R}_{-\alpha}(y)=O\left(\rho(y)^{-Q-\alpha}\right)
$$

as $y \rightarrow \infty$, and

$$
\left(u(x y)-u(x)-\omega(y)\left\langle\nabla_{\mathbb{G}} u(x), y\right\rangle\right) \widetilde{R}_{-\alpha}(y)=O\left(\rho(y)^{-Q+2-\alpha}\right)
$$

as $y \rightarrow 0$, since $([14,(1.37))$

$$
u(x y)-u(x)-\left\langle\nabla_{\mathbb{G}} u(x), y\right\rangle=O\left(\rho(y)^{2}\right)
$$

If $\varepsilon>0$, keeping in mind that both $\rho$ and $\widetilde{R}_{-\alpha}$ are check-invariant, we can write

$$
\int_{\rho\left(y^{-1} x\right)>\varepsilon}(u(y)-u(x)) \widetilde{R}_{-\alpha}\left(y^{-1} x\right) d y=\int_{\rho(y)>\varepsilon}(u(x y)-u(x)) \widetilde{R}_{-\alpha}(y) d y .
$$

Notice both integral are absolutely convergent, since $y \rightarrow(u(x y)-u(x)) \widetilde{R}_{-\alpha}(y)$ is a smooth function away from the origin and $(u(x y)-u(x)) \widetilde{R}_{-\alpha}(y)=$ $O\left(\rho(y)^{-Q-\alpha}\right)$ as $y \rightarrow \infty$. On the other hand, the map $y \rightarrow \omega(y)\left\langle\nabla_{\mathbb{G}} u(x), y\right\rangle \widetilde{R}_{-\alpha}(y)$ (that belongs to $L^{1}(\{\rho(y)>\varepsilon\})$ ) has zero integral, since $\omega(y) \widetilde{R}_{-\alpha}(y)$ is check-invariant, whereas $\left\langle\nabla_{\mathbb{G}} u(x), y^{-1}\right\rangle=\left\langle\nabla_{\mathbb{G}} u(x), y\right\rangle$. Therefore, we can write

$$
\begin{aligned}
\int_{\rho\left(y^{-1} x\right)>\varepsilon} & (u(y)-u(x)) \widetilde{R}_{-\alpha}\left(y^{-1} x\right) d y \\
= & \int_{\rho(y)>\varepsilon}\left(u(x y)-u(x)-\omega(y)\left\langle\nabla_{\mathbb{G}} u(x), y\right\rangle\right) \widetilde{R}_{-\alpha}(y) d y
\end{aligned}
$$

so that

$$
\int_{\mathbb{G}}\left(u(x y)-u(x)-\omega(y)\left\langle\nabla_{\mathbb{G}} u(x), y\right\rangle\right) \widetilde{R}_{-\alpha}(y) d y=\mathrm{P} \cdot \mathrm{V} \cdot \int_{\mathbb{G}}(u(y)-u(x)) \widetilde{R}_{-\alpha}\left(y^{-1} x\right) d y .
$$

We want to show now that

$$
\begin{aligned}
\int_{\rho\left(y^{-1} x\right)>\varepsilon} & (u(y)-u(x)) \widetilde{R}_{-\alpha}\left(y^{-1} x\right) d y \\
= & \int_{\rho\left(y^{-1} x\right)>\varepsilon} \mathcal{L} u(y) R_{2-\alpha}\left(x^{-1} y\right) d y+o(1)
\end{aligned}
$$

as $\varepsilon \rightarrow 0$. Notice both integrals absolutely converge at infinity. 
Take now $R>\varepsilon$. By Green identity (see e.g. 2], formula (5.43b)), we have

$$
\begin{aligned}
\int_{\varepsilon<\rho\left(y^{-1} x\right)<R} & (u(y)-u(x)) \widetilde{R}_{-\alpha}\left(y^{-1} x\right) d y \\
& =\int_{\varepsilon<\rho\left(y^{-1} x\right)<R}(u(y)-u(x)) \mathcal{L} R_{2-\alpha}\left(y^{-1} x\right) d y \\
& =\int_{\varepsilon<\rho\left(y^{-1} x\right)<R} \mathcal{L} u(y) R_{2-\alpha}\left(x^{-1} y\right) d y \\
& +\int_{\varepsilon=\rho\left(y^{-1} x\right)} R_{2-\alpha}\left(x^{-1} y\right) \sum_{j} X_{j}(u(y)-u(x))\left\langle X_{j}, \nu\right\rangle d \mathcal{H}^{n-1}(y) \\
& -\int_{\varepsilon=\rho\left(y^{-1} x\right)}(u(y)-u(x)) \sum_{j} X_{j} R_{2-\alpha}\left(x^{-1} y\right)\left\langle X_{j}, \nu\right\rangle d \mathcal{H}^{n-1}(y) \\
& +\int_{R=\rho\left(y^{-1} x\right)} R_{2-\alpha}\left(x^{-1} y\right) \sum_{j} X_{j}(u(y)-u(x))\left\langle X_{j}, \nu\right\rangle d \mathcal{H}^{n-1}(y) \\
& -\int_{R=\rho\left(y^{-1} x\right)}(u(y)-u(x)) \sum_{j} X_{j} R_{2-\alpha}\left(x^{-1} y\right)\left\langle X_{j}, \nu\right\rangle d \mathcal{H}^{n-1}(y) \\
& =\int_{\varepsilon<\rho\left(y^{-1} x\right)<R} \mathcal{L} u(y) R_{2-\alpha}\left(x^{-1} y\right) d y \\
& +I^{1}(\varepsilon)+I^{2}(\varepsilon)+J^{1}(R)+J^{2}(R),
\end{aligned}
$$

where $\nu$ in the outward unit normal to $\left\{\varepsilon<\rho\left(y^{-1} x\right)<R\right\}$. Obviously, $J_{1}$ vanishes as $R \rightarrow \infty$. Again, by Remark 3.2 , if $R$ is large, we have

$$
\begin{aligned}
& \left|J^{2}(R)\right| \leq C|u(x)| R^{1-\alpha-Q} \int_{R=\rho\left(y^{-1} x\right)} \sum_{j}\left|\left\langle X_{j}, \nu\right\rangle\right| d \mathcal{H}^{n-1}(y) \\
& \leq C|u(x)| R^{1-\alpha-Q} \int_{R=\rho\left(y^{-1} x\right)} d \mathcal{H}_{\mathbb{G}}^{Q-1}(y) \quad \text { (by Proposition 2.21) } \\
& =O\left(R^{-\alpha}\right),
\end{aligned}
$$

by (12). Thus we can take above the limit as $R \rightarrow \infty$ and we get

$$
\begin{aligned}
\int_{\varepsilon<\rho\left(y^{-1} x\right)} & (u(y)-u(x)) \widetilde{R}_{-\alpha}\left(y^{-1} x\right) d y \\
& =\int_{\varepsilon<\rho\left(y^{-1} x\right)} \mathcal{L} u(y) R_{2-\alpha}\left(x^{-1} y\right) d y+I^{1}(\varepsilon)+I^{2}(\varepsilon)
\end{aligned}
$$

(notice again both integrals are absolutely convergent).

Thus, (19) will follow by showing that $I^{1}(\varepsilon)+I^{2}(\varepsilon)=o(1)$ as $\varepsilon \rightarrow 0$.

Consider $I_{1}(\varepsilon)$. First of all, we notice that

$$
\begin{aligned}
\int_{\varepsilon=\rho\left(y^{-1} x\right)} R_{2-\alpha}\left(x^{-1} y\right)\left\langle X_{j}, \nu\right\rangle d \mathcal{H}^{n-1}(y) \\
=\int_{\varepsilon=\rho(y)} R_{2-\alpha}(y)\left\langle X_{j}, \nu\right\rangle d \mathcal{H}^{n-1}(y)=0
\end{aligned}
$$


for $j=1, \ldots, m$. Indeed we can write

$$
\begin{aligned}
=\int_{\varepsilon=\rho} & R_{2-\alpha}\left\langle X_{j}, \nu\right\rangle d \mathcal{H}^{n-1} \\
& =\int_{\varepsilon=\rho} R_{2-\alpha}\left(X_{j} R_{2-\alpha}\right)\left|\nabla R_{2-\alpha}\right|^{-1} d \mathcal{H}^{n-1},
\end{aligned}
$$

and (20) follows, since $\rho, R_{2-\alpha},\left|\nabla R_{2-\alpha}\right|$, and $\mathcal{H}^{n-1}$ are even under the change of variables $y \rightarrow{ }^{\mathrm{w}} y$, whereas $X_{j} R_{2-\alpha}$ is odd. Thus, by Proposition 2.2. we can write

$$
\begin{aligned}
& I_{1}(\varepsilon)=\int_{\varepsilon=\rho(y)} R_{2-\alpha}(y) \sum_{j}\left(X_{j} u\right)(x y)\left\langle X_{j}, \nu\right\rangle d \mathcal{H}^{n-1}(y) \\
& =\int_{\varepsilon=\rho(y)} R_{2-\alpha}(y) \sum_{j}\left[X_{j} u(x y)-X_{j} u(x)\right]\left\langle X_{j}, \nu\right\rangle d \mathcal{H}^{n-1}(y) \\
& \quad \leq C \max \left|X^{2} u\right| \varepsilon^{3-\alpha-Q} \int_{\varepsilon=\rho} d \mathcal{H}_{\mathbb{G}}^{Q-1}(y) \\
& \quad=O\left(\varepsilon^{2-\alpha}\right)=o(1) \quad \text { as } \varepsilon \rightarrow 0 .
\end{aligned}
$$

Finally, $I^{2}(\varepsilon)$ can be estimated by similar arguments. We write

$$
I^{2}(\varepsilon)=-\int_{\varepsilon=\rho}(u(x y)-u(x)) \sum_{j}\left(X_{j} R_{2-\alpha}\right)\left\langle X_{j}, \nu\right\rangle d \mathcal{H}^{n-1}(y),
$$

and we notice that, if $1 \leq \ell \leq m$

$$
\int_{\varepsilon=\rho} y_{\ell} \sum_{j}\left(X_{j} R_{2-\alpha}\right)\left\langle X_{j}, \nu\right\rangle d \mathcal{H}^{n-1}(y)=0 .
$$

Indeed, keeping again in mind Proposition 2.2, we have

$$
\begin{aligned}
\int_{\varepsilon=\rho} y_{\ell} & \sum_{j}\left(X_{j} R_{2-\alpha}\right)\left\langle X_{j}, \nu\right\rangle d \mathcal{H}^{n-1}(y) \\
= & \int_{\varepsilon=\rho} y_{\ell} \sum_{j}\left|X_{j} R_{2-\alpha}\right|^{2}\left|\nabla R_{2-\alpha}\right|^{-1} d \mathcal{H}^{n-1}(y) \\
= & \int_{\varepsilon=\rho} y_{\ell}\left|\nabla_{\mathbb{G}} R_{2-\alpha}\right|\left(\sum_{j}\left\langle X_{j}, \nu\right\rangle^{2}\right)^{1 / 2} d \mathcal{H}^{n-1}(y) \\
= & \int_{\varepsilon=\rho} y_{\ell}\left|\nabla_{\mathbb{G}} R_{2-\alpha}\right| d \mathcal{H}_{\mathbb{G}}^{Q-1}(y)=0
\end{aligned}
$$

since both $\left|\nabla_{\mathbb{G}} R_{2-\alpha}\right|$ and $\mathcal{H}_{\mathbb{G}}^{Q-1}$ are even with respect to the change of variable $y \rightarrow{ }^{\mathrm{w}} w$, whereas $y_{\ell}$ is odd.

Therefore, keeping in mind Taylor inequality in $\mathbb{G}$ (see, e.g. 14] Theorem 1.37), as well as Remark 3.2 and, again, Proposition 2.2, we can write

$$
\begin{aligned}
& \left|I^{2}(\varepsilon)\right| \\
& =\left|\int_{\varepsilon=\rho}\left(u(x y)-u(x)-\sum_{\ell} X_{\ell} u(x) y_{\ell}\right) \sum_{j}\left(X_{j} R_{2-\alpha}\right)\left\langle X_{j}, \nu\right\rangle d \mathcal{H}^{n-1}(y)\right| \\
& \quad=O\left(\varepsilon^{3-\alpha-Q}\right) \mathcal{H}_{\mathbb{G}}^{Q-1}(\{\varepsilon=\rho\})=O\left(\varepsilon^{2-\alpha}\right)=o(1) .
\end{aligned}
$$


This achieves the proof of (19). Taking the limit as $\varepsilon \rightarrow 0$ in (19), and keeping in mind that $R_{2-\alpha} \mathcal{L} u \in L^{1}(\mathbb{G})$, we get eventually

$$
\begin{aligned}
& \text { P.V } \int_{\mathbb{G}}(u(y)-u(x)) \widetilde{R}_{-\alpha}\left(y^{-1} x\right) d y=\int_{\mathbb{G}} \mathcal{L} u(y) R_{2-\alpha}\left(x^{-1} y\right) d y \\
& \quad=\mathcal{L}^{\alpha / 2} u,
\end{aligned}
$$

by Proposition 3.3. This achieves the proof of the theorem.

\section{MAin RESUlts}

Proposition 4.1 (see also Caffarelli \& Silvestre [5]). If $-\infty<\alpha<1$, the boundary value problem

$$
\left\{\begin{array}{l}
-t^{\alpha} \phi^{\prime \prime}+\phi=0 \\
\phi(0)=1 \\
\lim _{t \rightarrow+\infty} \phi(t)=0
\end{array}\right.
$$

has a solution $\phi \in \mathbf{C}^{2-\alpha}([0, \infty))$ of the form

$$
\phi(t)=c_{\alpha} t^{1 / 2} K_{1 / 2 k}\left(k^{-1} t^{k}\right),
$$

where $c_{\alpha}:=2^{1-1 / 2 k} \Gamma(1 / 2 k)^{-1} k^{-1 / 2 k}>0$ is a positive constant, $k=\frac{2-\alpha}{2}$, and $K_{1 / 2 k}$ is the modified Bessel function of second kind (see [37]). We know that

i) $0<\phi<1$. Moreover $\phi^{\prime}(t)$ has a finite limit as $t \rightarrow 0$ and, recursively,

$$
t^{\alpha+h-2} \phi^{(h)}(t) \text { has a finite limit as } t \rightarrow 0
$$

for $h=2,3, \ldots$

ii) $\phi^{\prime} \in L^{2}((0, \infty))$;

iii) $\phi(t)=c \sqrt{\frac{\pi k}{2}} t^{\alpha / 2} e^{-t^{k} / k}\left(1+O\left(\frac{1}{t}\right)\right)$ as $t \rightarrow \infty$;

iv) $\phi^{(h)}(t)=c_{h} t^{\alpha(1-h) / 2} e^{-t^{k} / k}(1+o(1))$ as $t \rightarrow \infty$ for $h=1,2, \ldots$.

Proof. By iteration, we can reduce ourselves to prove the assertion for $h=1$. Since $\phi$ is convex, $\phi^{\prime}(t) \rightarrow 0$ as $t \rightarrow \infty$ and we can write

$$
\phi^{\prime}(t)=\int_{t}^{\infty} s^{-\alpha} \phi(s) d s=c \sqrt{\frac{\pi k}{2}} \int_{t}^{\infty} s^{-\alpha / 2} e^{-s^{k} / k}(1+o(1)) d s .
$$

Then the estimate follows by the de l'Hôpital's rule.

Remark 4.2. The exact value of $\phi^{\prime}(0)$ can be explicitly computed keeping in mind that

$$
\phi^{\prime}(0)=c_{\alpha} \int_{0}^{\infty} s^{-\alpha+1 / 2} K_{1 / 2 k}\left(\frac{1}{k} s^{k}\right) d s=\frac{c_{\alpha}}{k} \int_{0}^{\infty} t^{(a+1) / 2} K_{1 / 2 k}\left(\frac{1}{k} t\right) d t,
$$

and that the last integral in turn can be explicitly evaluated by [22, 6.561 (16).

Put $\theta:=(1-a)^{a-1}$. If $u \in W^{1-a, 2}(\mathbb{G})$, for $y>0$ we set

$$
v(\cdot, y):=\phi\left(\theta y^{1-a} \mathcal{L}^{(1-a) / 2}\right) u:=\int_{0}^{\infty} \phi\left(\theta y^{1-a} \lambda^{(1-a) / 2}\right) d E(\lambda) u,
$$

Notice $v$ is well defined since $\phi$ is continuous and bounded in $[0, \infty)$. 
Choose now

$$
\alpha=-\frac{2 a}{1-a} .
$$

Proposition 4.3. Set $\Sigma_{+}=\mathbb{G}_{x} \times(0,1)_{y}$ and $\Sigma_{+}^{\varepsilon}=\mathbb{G}_{x} \times(\varepsilon, 1)_{y}$. If

$$
s \geq 1-\frac{a+1}{2} \quad \text { and } \quad u \in W^{s, 2}(\mathbb{G}),
$$

then $v \in W_{\widehat{\mathbb{G}}}^{1,2}\left(\Sigma_{+} ; y^{a} d x d y\right)$ and

$$
\|v\|_{W_{\hat{G}}^{1,2}\left(\Sigma_{+} ; y^{a} d x d y\right)} \leq C\|u\|_{W^{s, 2}(\mathbb{G})} .
$$

Moreover, if

$$
s \geq 2-\frac{a+1}{2} \quad \text { and } \quad u \in W^{s, 2}(\mathbb{G}),
$$

then $v \in W_{\hat{G}}^{2,2}\left(\Sigma_{+}^{\varepsilon} ; y^{a} d x d y\right)$ for any $\varepsilon>0$.

Proof. The function $v$ belongs to $L^{2}\left(\Sigma_{+} ; y^{a} d x d y\right)$. Indeed

$$
\begin{aligned}
& \|v\|_{L^{2}\left(\Sigma_{+} ; y^{a} d x d y\right)}^{2}=\int_{0}^{1} d y y^{a}\|v(y, \cdot)\|_{L^{2}\left(\mathbb{R}^{n}\right)}^{2} \\
& \quad=\int_{0}^{1} d y y^{a} \int_{0}^{\infty} \phi^{2}\left(\theta y^{1-a} \lambda^{(1-a) / 2}\right) d\|E(\lambda) u\|^{2} \leq C\|u\|_{L^{2}(\mathbb{G})}^{2},
\end{aligned}
$$

since $\phi$ is bounded.

On the other hand, if $\varepsilon \geq 0$,

$$
\begin{aligned}
\|v\|_{W_{\tilde{G}}^{k, 2}\left(\Sigma_{+} ; y^{a} d x d y\right)}^{2}=\sum_{0 \leq h \leq k} \sum_{|\beta| \leq k-h}\left\|\partial_{y}^{k} X^{\beta} v\right\|_{L^{2}\left(\Sigma_{+} ; y^{a} d x d y\right)}^{2} \\
=\sum_{0 \leq h \leq k} \int_{\varepsilon}^{1} d y y^{a} \int_{\mathbb{G}} d x \sum_{|\beta| \leq k-h}\left\|X^{\beta} \partial_{y}^{h} v\right\|^{2} \\
=\sum_{0 \leq h \leq k} \int_{\varepsilon}^{1} d y y^{a}\left\|\partial_{y}^{h} v\right\|_{W^{k-h, 2}(\mathbb{G})}^{2} \\
\approx \sum_{0 \leq h \leq k} \int_{\varepsilon}^{1} d y y^{a} \int_{\mathbb{G}} d x\left\|\mathcal{L}^{(k-h) / 2} \partial_{y}^{h} v\right\|_{W^{k-h, 2}(\mathbb{G})}^{2} \\
=\sum_{0 \leq h \leq k} \int_{\varepsilon}^{1} d y y^{a} \int_{0}^{\infty} \lambda^{k-h}\left|\partial_{y}^{h} \phi\left(\theta y^{1-a} \lambda^{(1-a) / 2}\right)\right|^{2} d\|E(\lambda) u\|^{2} .
\end{aligned}
$$

Recalling that

$$
\sum_{j=1}^{h} m_{j}=m
$$

and

$$
\sum_{j=1}^{h} j m_{j}=h
$$

the last term can be estimated by a sum of terms of the form

$$
\int_{\varepsilon}^{1} d y y^{a} \int_{0}^{\infty} \lambda^{k-h+(1-a) m} y^{2 m(1-a)-2 h}\left|\phi^{(h)}\left(\theta y^{1-a} \lambda^{(1-a) / 2}\right)\right|^{2} d\|E(\lambda) u\|^{2},
$$


with $m \leq h$. If we put $y \sqrt{\lambda}=\tau$, the last term is estimated by

$$
\begin{aligned}
\int_{0}^{\infty} d\|E(\lambda) u\|^{2} \lambda^{-\frac{a}{2}+k-h+(1-a) m-(1-a) m+h-\frac{1}{2}} \\
\quad \cdot \int_{\varepsilon \sqrt{\lambda}}^{\infty} \tau^{2 m(1-a)-2 h+2 a}\left|\phi^{(h)}\left(\theta \tau^{1-a}\right)\right|^{2} d\left(\tau^{1-a}\right) \\
=\int_{0}^{\infty} d\|E(\lambda) u\|^{2} \lambda^{k-\frac{a+1}{2}} \\
\quad \cdot \int_{(\varepsilon \sqrt{\lambda})^{1-a}}^{\infty} s^{2 m-2(h-a) /(1-a)}\left|\phi^{(h)}(\theta s)\right|^{2} d s
\end{aligned}
$$

Consider now the case $k=1$ (and therefore $h=m=1$, since the case $h=0$ yields the $L^{2}$-estimate we have already proved). Then we can take $\varepsilon=0$ and the last term becomes

$$
\begin{aligned}
\int_{0}^{\infty} & d\|E(\lambda) u\|^{2} \lambda^{1-\frac{a+1}{2}} \cdot \int_{0}^{\infty}\left|\phi^{\prime}(\theta s)\right|^{2} d s \\
& \leq \int_{0}^{\infty} d\|E(\lambda) u\|^{2}\left(1+\lambda^{s}\right) \cdot \int_{0}^{\infty}\left|\phi^{\prime}(\theta s)\right|^{2} d s \leq C\|u\|_{W^{s, 2}(\mathbb{G})}^{2},
\end{aligned}
$$

by ii) above.

Consider now the case $k=2$. In this case, we take $\varepsilon>0$ and we split the last integral in (24) as

$$
\int_{0}^{1} d\|E(\lambda) u\|^{2} \cdots+\int_{1}^{\infty} d\|E(\lambda) u\|^{2} \cdots:=I_{1}+I_{2} .
$$

Obviously,

$$
I_{2} \leq \int_{1}^{\infty} d\|E(\lambda) u\|^{2} \int_{\varepsilon^{1-a}}^{\infty} s^{2 m-2(h-a) /(1-a)}\left|\phi^{(h)}(\theta s)\right|^{2} d s<\infty,
$$

since $\phi^{(h)}(s)$ vanishes exponentially as $s \rightarrow \infty$. Analogously,

$$
\begin{aligned}
I_{1} \leq \int_{0}^{1} d\|E(\lambda) u\|^{2} \lambda^{2-\frac{a+1}{2}} \int_{(\varepsilon \sqrt{\lambda})^{1-a}}^{1} \cdots d s & \\
& +\int_{0}^{1} d\|E(\lambda) u\|^{2} \lambda^{2-\frac{a+1}{2}} \int_{1}^{\infty} \cdots d s
\end{aligned}
$$

Clearly, the second term in (25) is finite, again since since $\phi^{(h)}(s)$ vanishes exponentially as $s \rightarrow \infty$. Thus, we are reduced to estimate

$$
\begin{gathered}
\int_{0}^{1} d\|E(\lambda) u\|^{2} \lambda^{2-\frac{a+1}{2}} \\
\cdot \int_{(\varepsilon \sqrt{\lambda})^{1-a}}^{1} s^{2 m-2(h-a) /(1-a)-2 \alpha-2 h+4}\left|s^{\alpha+h-2} \phi^{(h)}(\theta s)\right|^{2} d s \\
\leq C \int_{0}^{1} d\|E(\lambda) u\|^{2} \lambda^{2-\frac{a+1}{2}} \\
\cdot \int_{(\varepsilon \sqrt{\lambda})^{1-a}}^{1} s^{2 m-2(h-a) /(1-a)-2 \alpha-2 h+4} d s,
\end{gathered}
$$


by Proposition 4.1, i). If we keep in mind that

$$
\int_{0}^{1} d\|E(\lambda) u\|^{2} \lambda^{2-\frac{a+1}{2}}<\infty
$$

since $u \in W_{\mathbb{G}}^{s, 2}(\mathbb{G})$, with $s \geq 2-\frac{a+1}{2}$, to achieve the proof of the proposition we have but to show that

$$
\begin{gathered}
2-\frac{a+1}{2}+(1-a)(2 m-2(h-a) /(1-a)-2 \alpha-2 h+5) \\
=(m-h)(1-a)-h+4 \geq(m-h)(1-a)+2>0 .
\end{gathered}
$$

On the other hand, if $h=1$, then necessarily $m=1$, so that $(m-h)(1-$ a) $+2=2$, whereas, if $h=2$, then either $m=1$ or $m=2$. In the first case $(m-h)(1-a)+2=a+1>0$. Finally, if $m=2$, then $(m-h)(1-a)+2=2$, achieving the proof of the proposition.

Theorem 4.4 (generalized subordination identity). If $u \in L^{2}(\mathbb{G})$ and $y>0$, we set

$$
v(\cdot, y):=\phi\left(\theta y^{1-a} \mathcal{L}^{(1-a) / 2}\right) u:=\int_{0}^{\infty} \phi\left(\theta y^{1-a} \lambda^{(1-a) / 2}\right) d E(\lambda) u,
$$

where $\theta:=(1-a)^{a-1}$ (we remind that $\phi$ is bounded, and therefore $v \in L^{2}(\mathbb{G})$ for $y>0)$.

We denote by $h(t, \cdot)$ the heat kernel associated with $-\mathcal{L}$ as in [13, and by $P_{\mathbb{G}}(\cdot, y)$ the "Poisson kernel"

$$
P_{\mathbb{G}}(\cdot, y):=C_{a} y^{1-a} \int_{0}^{\infty} t^{(a-3) / 2} e^{-\frac{y^{2}}{4 t}} h(t, \cdot) d t,
$$

where

$$
C_{a}=\frac{2^{a-1}}{\Gamma((1-a) / 2)}
$$

Then

$$
P_{\mathbb{G}}(\cdot, y) \geq 0
$$

by [24], [13], Theorem 3.1, and

$$
v(\cdot, y)=u * P_{\mathbb{G}}(\cdot, y) .
$$

Proof. By identity (8), p. 182 of [37, if $\nu>0$ and $z>0$, we can write

$$
K_{\nu}(z)=\frac{1}{2} \int_{0}^{\infty} \xi^{-\nu-1} e^{-\frac{1}{2} z\left(\xi+\frac{1}{\xi}\right)} d \xi .
$$

Then (keeping also in mind the definition of $\theta$ )

$$
\begin{aligned}
\phi(\theta z) & :=\frac{1}{2} c_{\alpha} \theta^{1 / 2} z^{1 / 2} \int_{0}^{\infty} \xi^{-\frac{1}{2 k}-1} e^{-\frac{\theta^{k}}{2 k} z^{k}\left(\xi+\frac{1}{\xi}\right)} d \xi \\
& =\frac{1}{2} c_{\alpha} \theta^{1 / 2} z^{1 / 2} \int_{0}^{\infty} \xi^{-\frac{1}{2 k}-1} e^{-\frac{\theta^{k}}{2 k} z^{k} \xi} e^{-\frac{\theta^{k}}{2 k} \frac{z^{k}}{\xi}} d \xi \\
& \left.=2^{(a-3) / 2} c_{\alpha} z^{1 / 2} \theta^{1 / 2} \int_{0}^{\infty} \tau^{(a-3) / 2} e^{-\tau z^{k}} e^{-\frac{z^{k}}{4 \tau}} d \tau \quad \text { (putting } \frac{\theta^{k}}{2 k} \xi=\tau\right) .
\end{aligned}
$$


Hence

$$
\begin{aligned}
\phi\left(\theta \lambda^{\frac{1-a}{2}} y^{1-a}\right) & =2^{(a-3) / 2} c_{\alpha} \lambda^{\frac{1-a}{4}} y^{(1-a) / 2} \theta^{1 / 2} \int_{0}^{\infty} \tau^{(a-3) / 2} e^{-\tau \sqrt{\lambda} y} e^{-\frac{\sqrt{\lambda} y}{4 \tau}} d \tau \\
& =2^{(a-3) / 2} c_{\alpha} \theta^{1 / 2} y^{1-a} \int_{0}^{\infty} t^{(a-3) / 2} e^{-\lambda t} e^{-\frac{y^{2}}{4 t}} d t
\end{aligned}
$$

putting $y \tau=\sqrt{\lambda} t$. In other words, $\lambda \rightarrow \phi\left(\theta \lambda^{\frac{1-a}{2}} y^{1-a}\right)$ is, up to a multiplicative constant, the Laplace transform of $t \rightarrow t^{(a-3) / 2} e^{-\frac{y^{2}}{4 t}}$.

For sake of brevity we set $C_{a}:=2^{(a-3) / 2} c_{\alpha} \theta^{1 / 2}$ (we remind that $\alpha$ depends on $a$ ). Thus we can write now

$$
\begin{aligned}
v(\cdot, y) & =C_{a} y^{1-a} \int_{0}^{\infty}\left(\int_{0}^{\infty} t^{(a-3) / 2} e^{-\lambda t} e^{-\frac{y^{2}}{4 t}} d t\right) d E(\lambda) u \\
& =C_{a} y^{1-a} \int_{0}^{\infty} t^{(a-3) / 2} e^{-\frac{y^{2}}{4 t}}\left(\int_{0}^{\infty} e^{-\lambda t} d E(\lambda) u\right) d t \\
& =C_{a} y^{1-a} \int_{0}^{\infty} t^{(a-3) / 2} e^{-\frac{y^{2}}{4 t}} u * h(t, \cdot) d t=u * P_{\mathbb{G}}(\cdot, y) .
\end{aligned}
$$

Remark 4.5. Formulas (27) and (26) make possible to give a different and more explicit representation of the lifting $v$ of $u$. On the other hand, the estimates of $h(t, \cdot)$ proved in [13] and [14] yield analogous estimates for $P_{\mathbb{G}}$. Indeed, if $I$ is a multi-index, then, if $\rho:=\rho(x)$,

$$
\begin{aligned}
\left|X^{I} P_{\mathbb{G}}(x, y)\right| & \leq C \int_{0}^{\infty} t^{(a-3) / 2}\left|X^{I} h(t, x)\right| d t \\
& =C \rho^{a-1} \int_{0}^{\infty} \tau^{(a-3) / 2}\left|X^{I} h\left(\tau \rho^{2}, x\right)\right| d \tau
\end{aligned}
$$

By 14, identity (1.73), we write now

$$
X^{I} h\left(\tau \rho^{2}, x\right)=(\sqrt{\tau} \rho)^{-Q-d(I)}\left|X^{I} h\left(1, \frac{x}{\sqrt{\tau} \rho}\right)\right|,
$$

and we notice that, since $h(1, \cdot) \in \mathcal{S}(\mathbb{G})$ (by [14, Proposition 1.74), if $N>0$, then

$$
\left|X^{I} h\left(1, \frac{x}{\sqrt{\tau} \rho}\right)\right| \leq C\left(1+\frac{1}{\sqrt{\tau}}\right)^{-N} \leq C \frac{\tau^{N / 2}}{1+\tau^{N / 2}} .
$$

Thus, eventually,

$$
\begin{aligned}
\left|X^{I} P_{\mathbb{G}}(x, y)\right| & \leq C \rho^{a-1-Q-d(I)} \int_{0}^{\infty} \tau^{(a-3-Q-d(I)) / 2} \frac{\tau^{N / 2}}{1+\tau^{N / 2}} \cdot d \tau \\
& \leq C \rho^{a-1-Q-d(I)}
\end{aligned}
$$

for large $\rho$. Then the lifting convolution $u * P_{\mathbb{G}}$ is well defined as long as $u(x)$ does not grow too fast as $x \rightarrow \infty$. We refer to [5] for similar growth conditions in the Euclidean setting. 
Moreover, if $u$ is sufficiently smooth,

$$
\begin{aligned}
y^{a} & \frac{v(x, y)-v(x, 0)}{y}=y^{a} \frac{u * P_{\mathbb{G}}(\cdot, y)-u(x)}{y} \\
= & \left(C_{a} \int_{0}^{\infty} t^{(a-3) / 2} e^{-\frac{y^{2}}{4 t}} u * h(t, \cdot) d t\right. \\
& \left.-C_{a} u(x) \int_{\mathbb{G}} \int_{0}^{\infty} t^{(a-3) / 2} e^{-\frac{y^{2}}{4 t}} h\left(t, \xi^{-1} x\right) d t d \xi\right) \\
= & C_{a} \int_{\mathbb{G}} \int_{0}^{\infty} t^{(a-3) / 2} e^{-\frac{y^{2}}{4 t}} h\left(t, \xi^{-1} x\right) d t(u(\xi)-u(x)) d \xi
\end{aligned}
$$

On the other hand

$$
\lim _{y \rightarrow 0^{+}} C_{a} \int_{0}^{\infty} t^{(a-3) / 2} e^{-\frac{y^{2}}{4 t}} h\left(t, \xi^{-1} x\right) d t=\tilde{C}_{a} \tilde{R}_{a-1} .
$$

Thus

$$
\lim _{y \rightarrow 0^{+}} y^{a} \frac{v(x, y)-v(x, 0)}{y}=C_{a} \int_{\mathbb{G}}(u(\xi)-u(x)) \tilde{R}_{a-1}(\xi) d \xi=\tilde{C}_{a} \mathcal{L}^{\frac{1-a}{2}} u(x) .
$$

Theorem 4.6. Let $u \in W_{\mathbb{G}}^{1-a, 2}(\mathbb{G})$ be given, $u \geq 0$, and assume $\mathcal{L}^{(1-a) / 2} u=$ 0 in an open set $\Omega$. With the notations of Theorem 4.4, we denote by $\hat{v}$ the function on $\hat{\mathbb{G}}$ obtained continuing $v$ by parity across $y=0$. Then

i) $\hat{v} \geq 0$;

ii) $\hat{v} \in W_{\widehat{\mathbb{G}}, \text { loc }}^{1,2}\left(\hat{\Omega} ; y^{a} d x d y\right)$, where $\hat{\Omega}:=\Omega \times(-1,1)$;

iii) $\hat{v}$ is a weak solution of the equation

$$
\operatorname{div}_{\widehat{\mathbb{G}}}\left(|y|^{a} \nabla_{\widehat{\mathbb{G}}} v\right)=0 \quad \text { in } \hat{\Omega} .
$$

Proof. Statement i) follows from previous Theorem 4.4.

The proofs of ii) and iii) are divided in several steps.

Step 1. From now on, we write $\Sigma_{-}:=\mathbb{G} \times(-1,0)$ and $\Sigma_{-}^{\varepsilon}:=\mathbb{G} \times(-1,-\varepsilon)$. If $\eta>0$, we set

$$
u_{\eta}:=(1+\eta \mathcal{L})^{-1} u:=\int_{0}^{\infty}(1+\eta \lambda)^{-1} d E(\lambda) u
$$

Then $u_{\eta} \in W_{\mathbb{G}}^{3-a, 2}(\mathbb{G})$ so that, with the notation of (22), by Proposition 4.3 . $\hat{v}_{\eta} \in W_{\widehat{\mathbb{G}}, \mathrm{loc}}^{2,2}\left(\Sigma_{ \pm} ; y^{a} d x d y\right)$. Moreover, just performing computations, we see that

$$
\operatorname{div}_{\widehat{\mathbb{G}}}\left(|y|^{a} \nabla_{\widehat{\mathbb{G}}} \hat{v}_{\eta}\right)=0
$$

in $\Sigma_{ \pm}$. Moreover, if $\psi \in \mathcal{D}\left(\Sigma_{ \pm}\right)$, then

$$
\int_{\Sigma_{ \pm}^{\varepsilon}}\left\langle\nabla_{\widehat{\mathbb{G}}} \hat{v}_{\eta}, \nabla_{\widehat{\mathbb{G}}} \psi\right\rangle|y|^{a} d x d y=0
$$

Step 2. The function $\hat{v}$ belongs to both $W_{\widehat{\mathbb{G}}}^{1,2}\left(\Sigma_{ \pm} ; y^{a} d x d y\right)$ (by Proposition 4.3) and in addition

$$
\int_{\Sigma_{ \pm}^{\varepsilon}}\left\langle\nabla_{\mathbb{G}} \hat{v}, \nabla_{\hat{\mathbb{G}}} \psi\right\rangle|y|^{a} d x d y=0
$$


for any $\psi \in \mathcal{D}\left(\hat{\Sigma}_{ \pm}\right)$. Indeed, by (23) $W_{\mathbb{G}}^{1-a, 2}(\mathbb{G})$. Indeed

$$
\left\|u_{\eta}-u\right\|_{W_{\mathbb{G}}^{1-a, 2}(\mathbb{G})}^{2}=\int_{0}^{\infty} \lambda^{1-a}\left|(1+\eta \lambda)^{-1}-1\right|^{2} d\|E(\lambda) u\|^{2} \rightarrow 0
$$

as $\eta \rightarrow 0$, by dominated convergence theorem. Since the function $y \rightarrow$ $|y|^{a}$ is smooth away from $\{y=0\}$, then $\hat{v}_{\eta}$ is smooth in $\Sigma_{ \pm}$, by classical Hörmander's theorem ([23]).

We notice that this argument going through regularization, equation in non-divergence form, integration by parts and variational equation is required by our abstract arguments that hides the divergence structure of the equation.

Step 3. Because of the properties of $A_{2}$-weights, $\hat{v} \in W_{\widehat{\mathbb{G}}, \mathrm{loc}}^{1,1}\left(\Sigma_{ \pm}\right) \cap L_{\text {loc }}^{1}(\Sigma)$. Moreover, with an obvious meaning of symbols,

$$
X_{j} \hat{v}=\widehat{X_{j} v} \quad \text { in } \Sigma, \text { for } j=1, \ldots, m
$$

and

$$
\partial_{y} \hat{v}= \pm \widehat{\partial_{y} v} \quad \text { in } \Sigma_{ \pm} .
$$

Clearly, this yields $\hat{v} \in W_{\widehat{\mathbb{G}}, \text { loc }}^{1,2}\left(\Sigma ; y^{a} d x d y\right)$ and therefore ii) holds. Now, (30) is obvious. As for (31), if $\psi \in \mathcal{D}(\hat{\Omega})$, by divergence theorem

$$
\begin{gathered}
\int_{\Sigma} \hat{v}\left(\partial_{y} \psi\right) d x d y=\lim _{\varepsilon \rightarrow 0} \int_{\Sigma_{+}^{\varepsilon}} \hat{v}\left(\partial_{y} \psi\right) d x d y+\lim _{\varepsilon \rightarrow 0} \int_{\Sigma_{-}^{\varepsilon}} \hat{v}\left(\partial_{y} \psi\right) d x d y \\
\quad=\lim _{\varepsilon \rightarrow 0} \int_{\Omega} v(\cdot, \varepsilon) \psi(\cdot, \varepsilon) d x-\lim _{\varepsilon \rightarrow 0} \int_{\Omega} v(\cdot,-\varepsilon) \psi(\cdot, \varepsilon) d x \\
\quad-\lim _{\varepsilon \rightarrow 0} \int_{\Sigma_{+}^{\varepsilon}}\left(\partial_{y} v\right) \psi d x d y+\lim _{\varepsilon \rightarrow 0} \int_{\Sigma_{-}^{\varepsilon}}\left(\widehat{\partial_{y} v}\right) \psi d x d y .
\end{gathered}
$$

Since $\hat{v}$ is locally Hölder continuous up to $y=0$

$$
\lim _{\varepsilon \rightarrow 0} \int_{\Omega} v(\cdot, \varepsilon) \psi(\cdot, \varepsilon) d x-\lim _{\varepsilon \rightarrow 0} \int_{\Omega} v(\cdot,-\varepsilon) \psi(\cdot, \varepsilon) d x=0
$$

and the assertion follows.

Step 4. By divergence theorem, if $\varepsilon \in(0,1)$ and $\psi \in \mathcal{D}(\hat{\Omega})$, then

$$
\int_{\Sigma_{ \pm}^{\varepsilon}}\left\langle\nabla_{\hat{\mathbb{G}}} \hat{v}, \nabla_{\widehat{\mathbb{G}}} \psi\right\rangle|y|^{a} d x d y=\int_{\Omega} \varepsilon^{a} \partial_{y} \hat{v}(x, \pm \varepsilon) \psi(x, \pm \varepsilon) d x
$$

Take now the limit as $\varepsilon \rightarrow 0$. Clearly

$$
\int_{\Sigma_{ \pm}^{\varepsilon}}\left\langle\nabla_{\widehat{\mathbb{G}}} \hat{v}, \nabla_{\hat{\mathbb{G}}} \psi\right\rangle|y|^{a} d x d y \rightarrow \int_{\hat{\Omega}}\left\langle\nabla_{\hat{\mathbb{G}}} \hat{v}, \nabla_{\hat{\mathbb{G}}} \psi\right\rangle|y|^{a} d x d y
$$

as $\varepsilon \rightarrow 0$. If we show that

$$
\varepsilon^{a} \partial_{y} \hat{v}(x, \pm \varepsilon) \rightarrow(1-a)^{a} \phi^{\prime}(0) \mathcal{L}^{\frac{1-a}{2}} u \quad \text { in } L^{2}(\mathbb{G}),
$$

then assertion iii) follows since $\mathcal{L}^{\frac{1-a}{2}} u$ vanishes on $\operatorname{supp} \psi$. 
To prove (34), we write

$$
\begin{aligned}
& \left\|\varepsilon^{a} \partial_{y} \hat{v}(x, \pm \varepsilon)-\phi^{\prime}(0) \mathcal{L}^{\frac{1-a}{2}} u\right\|_{L^{2}(\mathbb{G})}^{2} \\
& \quad=(1-a)^{2 a} \int_{0}^{\infty}\left|\phi^{\prime}\left(\theta \lambda^{\frac{1-a}{2}} \varepsilon^{1-a}\right)-\phi^{\prime}(0)\right|^{2} \lambda^{1-a} d\|E(\lambda) u\|^{2}
\end{aligned}
$$

and the assertion follows since $\phi^{\prime}$ is bounded.

This achieves the proof of the theorem.

Remark 4.7. By Theorem 2.9, $\hat{v}$ is locally Hölder continuous, and hence its trace $\hat{v}(\cdot, 0)$ on $\{y=0\}$ is well defined and it is straightforward to see that $v(\cdot, 0)=u$.

On the other hand, by a classical interpolation theorem ([27], Theorem 10.1), if $\hat{v}$ belongs to $W_{\mathbb{G}, \text { loc }}^{1,2}\left(\hat{\Omega} ; y^{a} d x d y\right)$, then its trace $u$ belongs to $W_{\mathbb{G}, \text { loc }}^{1-a, 2}(\Omega)$. This shows that our assumption $u \in W_{\mathbb{G}}^{1-a, 2}(\mathbb{G})$ is optimal as long as we are concerned with local regularity.

Theorem 4.8. Let $-1<a<1$ and let $u \in W_{\mathbb{G}}^{1-a, 2}(\mathbb{G})$ be given, $u \geq 0$ on all of $\mathbb{G}$. Assume $\mathcal{L}^{(1-a) / 2} u=0$ in an open set $\Omega \subset \mathbb{G}$.

Then there exist $C, b>0$ (independent of $u$ ) such that the following invariant Harnack inequality holds:

$$
\sup _{B_{c}(x, r)} u \leq C \inf _{B_{c}(x, r)} u
$$

for any metric ball $B_{c}(x, r)$ such that $B_{c}(x, b r) \subset \Omega$.

Proof. Let $C, b$ be as in Theorem [11]. By Theorems 4.6, 11] and by Lemma 2.6. we have:

$$
\begin{aligned}
\sup _{B_{c}(x, r)} u & =\sup _{\hat{B}_{c}((x, 0), r) \cap\{y=0\}} \hat{v} \leq \sup _{\hat{B}_{c}((x, 0), r)} \hat{v} \leq C \inf _{\hat{B}_{c}((x, 0), r)} \hat{v} \\
\leq & \inf _{\hat{B}_{c}((x, 0), r) \cap\{y=0\}} \hat{v}=\inf _{B_{c}(x, r)} u .
\end{aligned}
$$

\section{REFERENCES}

[1] Krzysztof Bogdan. The boundary Harnack principle for the fractional Laplacian. Studia Math., 123(1):43-80, 1997.

[2] Andrea Bonfiglioli, Ermanno Lanconelli, and Francesco Uguzzoni. Stratified Lie groups and potential theory for their sub-Laplacians. Springer Monographs in Mathematics. Springer, Berlin, 2007.

[3] Jean-Michel Bony, Philippe Courrège, and Pierre Priouret. Semi-groupes de Feller sur une variété à bord compacte et problèmes aux limites intégro-différentiels du second ordre donnant lieu au principe du maximum. Ann. Inst. Fourier (Grenoble), 18(fasc. 2):369-521 (1969), 1968.

[4] Nicolas Bourbaki. Éléments de mathématique. XXVI. Groupes et algèbres de Lie. Chapitre 1: Algèbres de Lie. Actualités Sci. Ind. No. 1285. Hermann, Paris, 1960.

[5] Luis Caffarelli and Luis Silvestre. An extension problem related to the fractional Laplacian. Comm. Partial Differential Equations, 32(7-9):1245-1260, 2007.

[6] Luis A. Caffarelli, Sandro Salsa, and Luis Silvestre. Regularity estimates for the solution and the free boundary of the obstacle problem for the fractional Laplacian. Invent. Math., 171(2):425-461, 2008.

[7] A.-P. Calderón. Inequalities for the maximal function relative to a metric. Studia Math., 57(3):297-306, 1976. 
[8] Luca Capogna, Donatella Danielli, and Nicola Garofalo. The geometric Sobolev embedding for vector fields and the isoperimetric inequality. Comm. Anal. Geom., 2(2):203-215, 1994.

[9] Sun-Yung Alice Chang and María del Mar González. Fractional Laplacian in conformal geometry. Adv. Math., 226(2):1410-1432, 2011.

[10] Philippe Courrège. Sur la forme intégro-différentielle des opérateurs de $\mathbf{C}_{k}^{\infty}$ dans $\mathbf{C}$ satisfaisant au principe du maximum. In Séminaire de Théorie du Potentiel, dirigé par M. Brelot, G. Choquet et J. Deny, 1965/66, Tome 10, No. 1, pages 1-38. Secrétariat mathématique, Paris, 1967.

[11] Eugene B. Fabes, Carlos E. Kenig, and Raul P. Serapioni. The local regularity of solutions of degenerate elliptic equations. Comm. Partial Differential Equations, 7(1):77$116,1982$.

[12] Fausto Ferrari and Bruno Franchi. A local doubling formula for the harmonic measure associated with subelliptic operators and applications. Comm. Partial Differential Equations, 28(1-2):1-60, 2003.

[13] Gerald B. Folland. Subelliptic estimates and function spaces on nilpotent Lie groups. Ark. Mat., 13(2):161-207, 1975.

[14] Gerald B. Folland and Elias M. Stein. Hardy spaces on homogeneous groups, volume 28 of Mathematical Notes. Princeton University Press, Princeton, N.J., 1982.

[15] Bruno Franchi and Ermanno Lanconelli. Hölder regularity theorem for a class of linear nonuniformly elliptic operators with measurable coefficients. Ann. Scuola Norm. Sup. Pisa Cl. Sci. (4), 10(4):523-541, 1983.

[16] Bruno Franchi and Raul Serapioni. Pointwise estimates for a class of strongly degenerate elliptic operators: a geometrical approach. Ann. Scuola Norm. Sup. Pisa Cl. Sci. (4), 14(4):527-568, 1987.

[17] Bruno Franchi, Raul Serapioni, and Francesco Serra Cassano. Meyers-Serrin type theorems and relaxation of variational integrals depending on vector fields. Houston J. Math., 22(4):859-890, 1996.

[18] Bruno Franchi, Raul Serapioni, and Francesco Serra Cassano. On the structure of finite perimeter sets in step 2 Carnot groups. J. Geom. Anal., 13(3):421-466, 2003.

[19] Bruno Franchi, Raul Serapioni, and Francesco Serra Cassano. Regular hypersurfaces, intrinsic perimeter and implicit function theorem in Carnot groups. Comm. Anal. Geom., 11(5):909-944, 2003.

[20] Nicola Garofalo and Duy-Minh Nhieu. Isoperimetric and Sobolev inequalities for Carnot-Carathéodory spaces and the existence of minimal surfaces. Comm. Pure Appl. Math., 49(10):1081-1144, 1996.

[21] Maria del Mar González and Jinggang Tan. In progress, 2012.

[22] Israel S. Gradshteyn and Iosif M. Ryzhik. Table of integrals, series, and products. Academic Press [Harcourt Brace Jovanovich Publishers], New York, 1980. Corrected and enlarged edition edited by Alan Jeffrey, Incorporating the fourth edition edited by Yu. V. Geronimus [Yu. V. Geronimus] and M. Yu. Tseytlin [M. Yu. Tseĭtlin], Translated from the Russian.

[23] Lars Hörmander. Hypoelliptic second order differential equations. Acta Math., 119:147-171, 1967.

[24] Gilbert A. Hunt. Semi-groups of measures on Lie groups. Trans. Amer. Math. Soc., 81:264-293, 1956.

[25] Naum S. Landkof. Foundations of modern potential theory. Springer-Verlag, New York, 1972. Translated from the Russian by A. P. Doohovskoy, Die Grundlehren der mathematischen Wissenschaften, Band 180.

[26] Paul Lévy. Sur les intégrales dont les éléments sont des variables aléatoires indépendantes. Ann. Scuola Norm. Sup. Pisa Cl. Sci. (2), 3(3-4):337-366, 1934.

[27] J.-L. Lions and E. Magenes. Non-homogeneous boundary value problems and applications. Vol. I. Springer-Verlag, New York, 1972. Translated from the French by P. Kenneth, Die Grundlehren der mathematischen Wissenschaften, Band 181.

[28] Guozhen Lu. Weighted Poincaré and Sobolev inequalities for vector fields satisfying Hörmander's condition and applications. Rev. Mat. Iberoamericana, 8(3):367-439, 1992. 
[29] Valentino Magnani. Differentiability and area formula on stratified Lie groups. Houston J. Math., 27(2):297-323, 2001.

[30] Kyûya Masuda. Anti-locality of the one-half power of elliptic differential operators. Publ. Res. Inst. Math. Sci., 8:207-210, 1972.

[31] Benjamin Muckenhoupt. Weighted norm inequalities for the Hardy maximal function. Trans. Amer. Math. Soc., 165:207-226, 1972.

[32] Yannick Sire and Enrico Valdinoci. Fractional Laplacian phase transitions and boundary reactions: a geometric inequality and a symmetry result. J. Funct. Anal., 256(6):1842-1864, 2009.

[33] Guido Stampacchia. Le problème de Dirichlet pour les équations elliptiques du second ordre à coefficients discontinus. Ann. Inst. Fourier (Grenoble), 15(fasc. 1):189-258, 1965.

[34] Elias M. Stein. Harmonic analysis: real-variable methods, orthogonality, and oscillatory integrals, volume 43 of Princeton Mathematical Series. Princeton University Press, Princeton, NJ, 1993. With the assistance of Timothy S. Murphy, Monographs in Harmonic Analysis, III.

[35] Pablo Raúl Stinga and José Luis Torrea. Extension problem and Harnack's inequality for some fractional operators. Comm. Partial Differential Equations, 35(11):20922122, 2010.

[36] Pablo Raúl Stinga and Chao Zhang. Harnack's inequality for fractional nonlocal equations. arXiv:1203.1518, 2012.

[37] George N. Watson. A Treatise on the Theory of Bessel Functions. Cambridge University Press, Cambridge, England, 1944.

Fausto Ferrari and Bruno Franchi

Dipartimento di Matematica

Piazza di Porta S. Donato 5

40126 Bologna, Italy;

e-mail:

fausto.ferrari@unibo.it

bruno.franchi@unibo.it 